\title{
Uniqueness and Novel Finite-Time Stability of Solutions for a Class of Nonlinear Fractional Delay Difference Systems
}

\author{
Danfeng Luo and Zhiguo Luo \\ Department of Mathematics, Hunan Normal University, Changsha, Hunan 410081, China \\ Correspondence should be addressed to Zhiguo Luo; luozg1956@163.com
}

Received 5 July 2018; Revised 28 August 2018; Accepted 16 September 2018; Published 30 September 2018

Guest Editor: Ahmed S. Hendy

Copyright (C) 2018 Danfeng Luo and Zhiguo Luo. This is an open access article distributed under the Creative Commons Attribution License, which permits unrestricted use, distribution, and reproduction in any medium, provided the original work is properly cited.

\begin{abstract}
This paper focuses on the uniqueness and novel finite-time stability of solutions for a kind of fractional-order nonlinear difference equations with time-varying delays. Under some new criteria and by applying the generalized Gronwall inequality, the new constructive results have been established in the literature. As an application, two typical examples are delineated to demonstrate the effectiveness of our theoretical results.
\end{abstract}

\section{Introduction}

The time difference of fractional order was firstly studied by Kuttner [1] in 1957; since then, various kinds of definitions of fractional difference were studied by many authors. We know fractional difference equations play an important role in promoting modern mathematics development and have been widely applied, especially in physics, dynamic mechanics, medicines, and communications. There is a growing tendency nowadays that many experts show their great enthusiasm for fractional difference equations, and in the past few years, a lot of achievements have been done. For an extensive collection of such results, we recommend the readers to monograph [2] and papers [3-16].

The study about uniqueness of discrete solutions for fractional difference equations is one of the most interesting and valuable topics. Recently, Abdeljawad et al. [3] studied the following nonlinear fractional difference system

$$
\begin{aligned}
\left({ }_{a}^{C F R} \nabla^{\alpha} y\right)(t) & =f(t, y(t)), \quad t \in \mathbb{N}_{a, b}, \\
y(a) & =c .
\end{aligned}
$$

Let $f(t, y)$ satisfy Lipschitz condition: there exists a constant $A>0$ such that

$$
\left|f\left(t, y_{1}\right)-f\left(t, y_{2}\right)\right| \leq A\left|y_{1}-y_{2}\right|
$$

and $f: \mathbb{N}_{a, b} \times \mathbb{R} \longrightarrow \mathbb{R}$ and $y: \mathbb{N}_{a, b} \longrightarrow \mathbb{R}$. By Banach Contraction Principle, authors obtained that system (1) had a unique solution $x \in X, X=\left\{x: \max _{t \in \mathbb{N}_{a, b}}|x(t)|<\infty\right\}$, if

$$
\frac{A}{B(\alpha-1)}\left((2-\alpha)(b-a)+\frac{(\alpha-1)(b-a)^{\overline{2}}}{2}\right)<1,
$$

where $B(\alpha)$ is a normalization positive constant depending on $\alpha$ satisfying $B(0)=B(1)=1$, and ${ }_{a}^{C F R} \nabla^{\alpha}$ is a fractional operator defined as in Definition 4 of paper [3].

In [4], Abdeljawad and M. Al-Mdallal considered the fractional difference system

$$
\begin{aligned}
\left({ }_{a}^{A B C} \nabla^{\alpha} y\right)(t) & =f(t, y(t)), \quad t \in \mathbb{N}_{a, b}, \\
y(a) & =c,
\end{aligned}
$$

such that $b \equiv a(\bmod 1), f(a, y(a))=0$. Let $f$ admit the Lipschitz condition, $A$ be a Lipschitz constant, and $y$ : $\mathbb{N}_{a, b} \longrightarrow \mathbb{R}$. Then system (4) had a unique solution provided that

$$
A\left(\frac{1-\alpha}{B(\alpha)}++\frac{(b-a)^{\bar{\alpha}}}{\Gamma(\alpha) B(\alpha)}\right)<1
$$

where ${ }_{a}^{A B C} \nabla^{\alpha}$ is a fractional operator, and we can see it in Definition 4 of paper [4]. 
What will happen if the nonlinear equations (1) and (4) subjecting the initial value condition extend into fractional delay difference equation? We are particularly interested in fractional difference equation involving time-varying delays.

On the other hand, stability analysis is also one of the most crucial themes for fractional nonlinear systems, such as $[17,18]$ researching stability in nondelay fractional systems and $[14,19-22]$ in delay fractional difference systems. Specifically, in $[17,18]$, using Lyapunov's direct method, the stability of discrete nonautonomous systems with the nabla Caputo fractional difference was studied. In [19], the authors studied a class of linear fractional difference equations with impulse effects, and they provided the generalized Mittag-Leffler stability by numerical illustration. In papers [20,22], asymptotic stability of a fractional discrete system was discussed and the theorem for a discrete fractional Lyapunov direct method was proved. In [21], the researchers investigated the stability of the equilibrium solution of a linear fractional difference equation with the initial condition, and to achieve this target, the wellknown unilateral $z$-transform was successfully employed. In paper [14], the researchers considered the following linear fractional difference equations with a constant delay

$$
{ }^{C} \Delta_{a}^{v} x(t)=A_{0} x(t+v)+A_{1} x(t+v-k), \quad 0<v<1,
$$

where $x(t) \in \mathbb{R}^{l}, k$ is a fixed positive integer, and ${ }^{C} \Delta_{a}^{v} x(t)$ denotes the Caputo delta fractional difference of $x(t)$ on the discrete time. The finite-time stable conclusions are presented in the addressed paper.

Deeply inspired by $[3,4,14]$ and other mentioned papers, in this paper, we are concerned with the uniqueness and finite-time stability of solutions for the following fractional discrete equation with time-varying delays

$$
\begin{aligned}
{ }^{C} \Delta_{a}^{v} x(t)= & A x(t)+B x(t-h(t))+D w(t) \\
& +f(t, x(t), x(t-h(t)), w(t)), \\
x(t)= & \phi(t), \quad t \in J_{2},
\end{aligned}
$$

where ${ }^{C} \Delta_{a}^{v}$ denotes the Caputo fractional difference operator with $v \in(0,1]$, and $J_{1}=\{t \in \mathbb{Z} \mid a+1-v \leq t \leq a+M\}$, and $J_{2}=\{t \in \mathbb{Z} \mid a+1-v-h \leq t \leq a+1-v\}, a \in \mathbb{R}, M$ is a positive integer; $x(t) \in \mathbb{R}^{n}$ is the state vector, $w(t) \in \mathbb{R}^{m}$ is the disturbance vector, $h(t)$ is a function satisfying $0 \leq h(t) \leq h$, and $\phi(t) \in \mathbb{R}^{n}$ is the given function; $A \in \mathbb{R}^{n \times n}, B \in \mathbb{R}^{n \times n}, D \in$ $\mathbb{R}^{n \times m}$ are constant matrices, and $f: J_{1} \times \mathbb{R}^{n} \times \mathbb{R}^{n} \times \mathbb{R}^{m} \longrightarrow \mathbb{R}^{n}$.

Compared with some recent results in the literatures, such as $[14,17,19-22]$, the chief contributions of this study contain at least the following three:

(1) In $[14,17,19-22]$, the literatures investigated the stability of fractional difference equations with constant delays, but the delay term we studied in system (7) is a bounded function with respect to the variable $t$. This is a significant breakthrough in dealing with fractional difference system with time-varying delays.

(2) The model we are concerned with is more generalized, some ones in the articles are the special cases of it.
In $[19,22]$, the coefficients of the fractional discrete system researched by authors are one-dimensional real numbers which are too simple to describe the mathematical model well, and we adopt constant matrices as coefficients in (7). Therefore, the generalized models are originally discussed in the present paper. Furthermore, our conclusions can also be applied to the equations with function matrices, and you can see it by the following Corollaries 10 and 13 .

(3) An innovative method based on the generalized Gronwall inequality is exploited to discuss the uniqueness and finite-time stability of the solutions for the fractional-order difference equation with time-varying delays. The results established are essentially new.

The following article is organized as follows: in Section 2, we will recall some known results for our considerations. Some lemmas and definitions are useful to our work. Section 3 is devoted to researching the uniqueness of solutions for the fractional-order difference equation with time delay. Subsequently, we investigate the finite-time stability of the addressed equation, and then we will come up with the main theorem. To explain the results clearly, we finally provide two examples in Section 4.

\section{Preliminaries}

In this section, we plan to introduce some basic definitions and lemmas which are used throughout this paper.

Definition $1([23,24])$. We define

$$
t^{\underline{\nu}}:=\frac{\Gamma(t+1)}{\Gamma(t+1-v)},
$$

as for any $t$ and $v$ for which the right-hand side is defined. Here and in what follows $\Gamma$ denotes the gamma function. We also appeal to the common convention that if $t+1-v$ is a pole of the gamma function and $t+1$ is not a pole, then $t^{\underline{\nu}}=0$.

Definition 2 ([24]). The $v$-th fractional sum of a function $f$, for $v>0$, is defined to be

$$
\Delta_{a}^{-v} f(t)=\Delta_{a}^{-v} f(t ; a):=\frac{1}{\Gamma(\nu)} \sum_{s=a}^{t-v}(t-s-1) \frac{\nu-1}{v} f(s),
$$

where $t \in\{a+v, a+v+1, \ldots\}=,: \mathbb{N}_{a+v}$. We also define the $\nu$-th fractional difference, where $v>0$ and $0 \leq N-1<v \leq N$ with $N \in \mathbb{N}$, to be $\Delta^{\nu} f(t):=\Delta^{N} \Delta^{\nu-N} f(t)$, where $t \in \mathbb{N}_{a+\nu}$.

Lemma 3 ([15]). Assume that $\mu>0$ and $f$ is defined on $\mathbb{N}_{a}$. Then

$$
\begin{aligned}
\Delta^{-\mu} \Delta^{\mu} f(t) & =f(t)-\sum_{k=0}^{n-1} \frac{(t-a)^{(k)}}{k !} \Delta^{k} f(a) \\
& =f(t)+c_{0}+c_{1} t+\cdots+c_{n-1} t^{(n-1)},
\end{aligned}
$$

where $n$ is the smallest integer greater than or equal to $\mu, c_{i} \in \mathbb{R}$, $i=1,2, \cdots, n-1$. 
Lemma 4 ([10]). Let $v \in \mathbb{R}$ and $t, s \in \mathbb{R}$ such that $(t-s)^{\underline{v}}$ is well defined, then $\Delta_{s}(t-s)^{\underline{v}}=-v(t-s-1)^{\nu-1}$.

Definition 5 ([14]). Given positive numbers $c_{1}, c_{2}$ satisfying $c_{1}<c_{2}$, system (7) is finite-time stable if and only if

$$
\|\phi\| \leq c_{1} \Longrightarrow\|x(t)\| \leq c_{2}, \quad \forall t \in J_{1} \cup J_{2},
$$

for all disturbances $w(t)$ satisfying the following condition:

$$
\exists \eta>0: w^{T}(t) w(t) \leq \eta^{2} .
$$

Definition 6. A function $x(t)$ is called the solution of (7) if $x(t)$ satisfies

$$
x(t)= \begin{cases}x(a)+\frac{1}{\Gamma(\nu)} \sum_{s=a}^{t-\gamma}(t-s-1) \frac{\nu-1}{n}[A x(s)+B x(s-h(s))+D w(s)+f(s, x(s), x(s-h(s)), w(s))], & t \in J_{1}, \\ \phi(t), & t \in J_{2} .\end{cases}
$$

Lemma 7 (generalized Gronwall inequality). Let $\alpha>0$, and $u(t), v(t)$ be nonnegative functions and $w(t)$ be nonnegative, nondecreasing function for $t \in \mathbb{N}_{a}$ such that $w(t) \leq M$, where $M$ is a constant. If

$$
u(t) \leq v(t)+w(t) \Gamma(\alpha) \Delta_{a}^{-\alpha} u(t),
$$

then

$$
u(t) \leq v(t)+\sum_{k=1}^{\infty}(w(t) \Gamma(\alpha))^{k} \Delta_{a}^{-k \alpha} v(t) .
$$

Proof. Define operator

$$
B \phi(t)=w(t) \sum_{s=a}^{t-\alpha}(t-s-1) \frac{\alpha-1}{\underline{\alpha}} \phi(s),
$$

then from (14), we know

$$
u(t) \leq v(t)+B u(t),
$$

which implies that $u(t) \leq \sum_{k=0}^{n-1} B^{k} v(t)+B^{n} u(t)$. The following proof process is similar to the relevant conclusion, and we can refer to Theorem 3.2 in [14].

\section{Main Results}

Let $\lambda(A)$ be the set of all eigenvalues of $A$ and $\lambda_{\max }(A)=$ $\max \{\operatorname{Re}(\lambda): \lambda \in \lambda(A)\}$. Assume that $\|A\|$ denotes the spectral norm defined by $\sqrt{\lambda_{\max }\left(A^{T} A\right)}$, and let $\|x\|$ be the norm of $x(t)=\left(x_{1}(t), x_{2}(t), \cdots, x_{n}(t)\right)^{T} \in \mathbb{R}^{n}$ defined by $\|x\|=\max _{t \in J_{1} \cup J_{2}}\left(\sum_{i=1}^{n} x_{i}^{2}\right)^{1 / 2}$. Suppose that $\mathscr{B}^{+}\left(J_{1}\right)$ denotes the set of all nonnegative bounded functions on $J_{1}$. Assume that the nonlinear function $f: J_{1} \times \mathbb{R}^{n} \times \mathbb{R}^{n} \times \mathbb{R}^{m} \longrightarrow \mathbb{R}^{n}$ satisfies the condition $\left(H_{1}\right)$ : there exists a positive constant $l(t) \in \mathscr{B}^{+}\left(J_{1}\right)$ such that

$$
\begin{aligned}
& \left\|f\left(t, x_{1}, y_{1}, w_{1}\right)-f\left(t, x_{2}, y_{2}, w_{2}\right)\right\| \\
& \quad \leq l(t)\left(\left\|x_{1}-x_{2}\right\|+\left\|y_{1}-y_{2}\right\|+\left\|w_{1}-w_{2}\right\|\right),
\end{aligned}
$$

where $f(t, 0,0,0)=0$. In this section, we always assume that

$$
\begin{aligned}
\|A\| & =\bar{a}, \\
\|B\| & =\bar{b},
\end{aligned}
$$

$$
\begin{array}{r}
\|D\|=\bar{d} \\
\sup _{s \in[a, t-\nu]} l(s)=\bar{L} .
\end{array}
$$

Theorem 8. Assume that $0 \leq(\bar{a}+\bar{b}+2 \bar{L}) / \Gamma(\nu)<1$, then system (7) has a unique solution on $J_{1} \cup J_{2}$ if the condition $\left(H_{1}\right)$ holds.

Proof. Let $x(t)$ and $\widetilde{x}(t)$ be any two different solutions to system (7), then $x(t)$ and $\widetilde{x}(t)$ both satisfy (13). Let $z(t)=$ $x(t)-\widetilde{x}(t)$.

We can easily obtain $z(t)=0$ for $t \in J_{2}$. That is to say, system (7) has a unique solution as $t \in J_{2}$.

When $t \in J_{1}=J_{11} \cup J_{12}$, and $J_{11}=\{t \in \mathbb{Z} \mid a+1-v \leq t \leq$ $a+\nu\}, J_{12}=\{t \in \mathbb{Z} \mid a+\nu \leq t \leq a+M\}$, we get

$$
\begin{aligned}
z(t) & =\frac{1}{\Gamma(\nu)} \sum_{s=a}^{t-v}(t-s-1)^{\frac{\nu-1}{3}}[A z(s)+B z(s-h(s)) \\
& +f(s, x(s), x(s-h(s)), w(s)) \\
& -f(s, \widetilde{x}(s), \widetilde{x}(s-h(s)), w(s))] .
\end{aligned}
$$

If $t \in J_{11}$, then

$$
\begin{aligned}
z(t) & =\frac{1}{\Gamma(\nu)} \sum_{s=a}^{t-v}(t-s-1)^{\frac{\nu-1}{2}}[A z(s) \\
& +f(s, x(s), x(s-h(s)), w(s)) \\
& -f(s, \widetilde{x}(s), \widetilde{x}(s-h(s)), w(s))] .
\end{aligned}
$$

Now applying the norm $\|\cdot\|$ on both sides of (21), we get

$$
\begin{aligned}
& \|z(t)\| \leq \frac{1}{\Gamma(\nu)} \sum_{s=a}^{t-v}(t-s-1)^{\frac{v-1}{2}}[\|A\|\|z(s)\| \\
& +\| f(s, x(s), x(s-h(s)), w(s)) \\
& -f(s, \tilde{x}(s), \tilde{x}(s-h(s)), w(s)) \|] \leq \frac{1}{\Gamma(\nu)} \sum_{s=a}^{t-v}(t-s \\
& -1)^{\frac{\nu-1}{2}}[(\|A\|+l(s))\|z(s)\|] \leq \frac{\bar{a}+\bar{L}}{\Gamma(\nu)} \sum_{s=a}^{t-v}(t-s \\
& -1)^{\frac{\nu-1}{}}\|z(s)\| .
\end{aligned}
$$


Applying the Generalized Gronwall Inequality in Lemma 7, we have

$$
\|z(t)\| \leq 0
$$

Namely, $x(t)=\tilde{x}(t)$ for $t \in J_{11}$.

If $t \in J_{12}$, then applying the norm $\|\cdot\|$ on both sides of (20), it follows that

$$
\begin{gathered}
\|z(t)\| \leq \frac{1}{\Gamma(\nu)} \sum_{s=a}^{t-v}(t-s-1) \frac{v-1}{} \\
\cdot[(\bar{a}+l(s))\|z(s)\|+(\bar{b}+l(s))\|z(s-h(s))\|] \\
\leq \frac{\bar{a}+\bar{L}}{\Gamma(\nu)} \sum_{s=a}^{t-v}(t-s-1) \frac{v-1}{n}\|z(s)\|+\frac{\bar{b}+\bar{L}}{\Gamma(\nu)} \\
\cdot \sum_{s=a+v+1}^{t-v}(t-s-1)^{\frac{\nu-1}{x}} \times\|z(s-h(s))\| .
\end{gathered}
$$

Let $z^{*}(t)=\sup _{\theta \in[-h, 0]}\|z(t+\theta)\|, t \in J_{12}$, then we have

$$
z^{*}(t) \leq \frac{\bar{a}+\bar{b}+2 \bar{L}}{\Gamma(\nu)} \sum_{s=a}^{t-v}(t-s-1) \frac{v-1}{2} z^{*}(s) .
$$

Similarly, applying the generalized Gronwall inequality in Lemma 7, it follows that

$$
\|z(t)\| \leq z^{*}(t)<0 .
$$

Hence we can obtain $x(t)=\tilde{x}(t)$. This completes the proof.

Remark 9. When we demonstrate the uniqueness of solutions for the fractional discrete system with time delay, we find that the conditions we needed have nothing to do with the disturbance vector $w(t)$. That is to say, disturbance vector does not affect the uniqueness of solution for the system.

One can extend the constant matrices in (7) to the function form as follows:

$$
\begin{aligned}
{ }^{C} \Delta_{a}^{v} x(t)= & A(t) x(t)+B(t) x(t-h(t)) \\
& +D(t) w(t) \\
& +f(t, x(t), x(t-h(t)), w(t)), \\
x(t)= & \phi(t), \quad t \in J_{2} .
\end{aligned}
$$

Our conclusion (Theorem 8) can also be applied to (27) if $f(\cdot)$ satisfies the condition $\left(H_{1}\right)$. In the case, let $\|A(t)\|=\tilde{a}$, $\|B(t)\|=\widetilde{b},\|D(t)\|=\widetilde{d}$ in the same proof.

Corollary 10. Assume that $0 \leq(\widetilde{a}+\widetilde{b}+2 \bar{L}) / \Gamma(\nu)<1$, then system (27) has a unique solution on $J_{1} \cup J_{2}$ if the condition $\left(H_{1}\right)$ holds.

Theorem 11. Suppose that $\left(H_{1}\right)$ holds, and $0 \leq(\bar{a}+\bar{b}+$ $2 \bar{L}) / \Gamma(\nu)<1$, and there exist positive numbers $c_{1}<c_{2}$, and $\|\phi\| \leq c_{1}$, then system (7) is finite-time stable on $J_{1} \cup J_{2}$ if

$$
\begin{aligned}
& \left(c_{1}+\frac{\eta(\bar{d}+\bar{L}) M^{\underline{v}}}{\Gamma(\nu+1)}\right) \\
& \quad\left(1+\sum_{k=1}^{\infty}(\bar{a}+\bar{b}+2 \bar{L})^{k} \cdot \frac{M^{\underline{k v}}}{\Gamma(k \nu+1)}\right) \leq c_{2} .
\end{aligned}
$$

Proof. Let $u(t)=\sup _{\theta \in[a+1-v-h, t]}\|x(\theta)\|$, for $t \in J_{1}$. We have $\|x(s)\| \leq u(s)$, and $\|x(s-h(s))\| \leq u(s), \forall s \in[0, t]$. According to (12) and (13), one can obtain

$$
\begin{aligned}
& \|x(t)\| \leq\|x(a)\|+\frac{1}{\Gamma(\nu)} \sum_{s=a}^{t-\nu}(t-s-1) \frac{\nu-1}{2}[\|A\|\|x(s)\| \\
& +\|B\|\|x(s-h(s))\|+\|D\|\|w(s)\| \\
& +\|f(s, x(s), x(s-h(s)), w(s))\|] \leq\|\phi\| \\
& +\frac{\bar{a}+\bar{b}+2 \bar{L}}{\Gamma(\nu)} \sum_{s=a}^{t-v}(t-s-1) \frac{\nu-1}{u} u(s)+\frac{\eta(\bar{d}+\bar{L})}{\Gamma(\nu)} \\
& \cdot \frac{(t-a)^{\underline{v}}}{\nu} \leq\|\phi\|+\frac{\eta(\bar{d}+\bar{L}) M^{\underline{v}}}{\Gamma(\nu+1)}+\frac{\bar{a}+\bar{b}+2 \bar{L}}{\Gamma(\nu)} \\
& \cdot \sum_{s=a}^{t-v}(t-s-1) \frac{v-1}{v} u(s)
\end{aligned}
$$

As for all $\theta \in[a+1-v, t]$, we have

$$
\begin{aligned}
\|x(\theta)\| \leq & \|\phi\|+\frac{\eta(\bar{d}+\bar{L}) M^{\underline{\nu}}}{\Gamma(\nu+1)} \\
& +\frac{\bar{a}+\bar{b}+2 \bar{L}}{\Gamma(\nu)} \sum_{s=a}^{\theta-\nu}(\theta-s-1) \frac{\nu-1}{u} u(s) \\
\leq & \|\phi\|+\frac{\eta(\bar{d}+\bar{L}) M^{\underline{v}}}{\Gamma(\nu+1)} \\
& +\frac{\bar{a}+\bar{b}+2 \bar{L}}{\Gamma(\nu)} \sum_{s=a}^{t-v}(t-s-1)^{\frac{\nu-1}{u}} u(s) .
\end{aligned}
$$

Therefore, we have

$$
\begin{aligned}
u(t) & =\sup _{\theta \in[a+1-\nu-h, t]}\|x(\theta)\| \\
& \leq \max \left\{\sup _{\theta \in[a+1-\nu-h, a+1-v]}\|x(\theta)\|, \sup _{\theta \in[a+1-v, t]}\|x(\theta)\|\right\} \\
& \leq \max \left\{\|\phi\|,\|\phi\|+\frac{\eta(\bar{d}+\bar{L}) M^{\underline{v}}}{\Gamma(\nu+1)}\right. \\
& \left.+\frac{\bar{a}+\bar{b}+2 \bar{L}^{t-v}}{\Gamma(\nu)} \sum_{s=a}(t-s-1)^{\frac{\nu-1}{u}} u(s)\right\}=\|\phi\| \\
& +\frac{\eta(\bar{d}+\bar{L}) M^{\underline{v}}}{\Gamma(\nu+1)}+\frac{\bar{a}+\bar{b}+2 \bar{L}^{t-v}}{\Gamma(\nu)} \sum_{s=a}^{(t-s-1) \frac{\nu-1}{u}} u(s) .
\end{aligned}
$$


According to Lemma 7, we can get

$$
\begin{aligned}
u(t) & \leq\|\phi\|+\frac{\eta(\bar{d}+\bar{L}) M^{\underline{v}}}{\Gamma(\nu+1)}+\sum_{k=1}^{\infty}(\bar{a}+\bar{b}+2 \bar{L})^{k} \\
& \frac{1}{\Gamma(k v)} \sum_{s=a}^{t-k v}(t-s-1)^{\frac{k v-1}{}} \\
& \times\left(\|\phi\|+\frac{\eta(\bar{d}+\bar{L}) M^{\underline{v}}}{\Gamma(\nu+1)}\right) \\
& \leq\left(\|\phi\|+\frac{\eta(\bar{d}+\bar{L}) M^{\underline{v}}}{\Gamma(\nu+1)}\right) \\
& \cdot\left(1+\sum_{k=1}^{\infty}(\bar{a}+\bar{b}+2 \bar{L})^{k} \cdot \frac{M^{\frac{k v}{v}}}{\Gamma(k v+1)}\right) .
\end{aligned}
$$

Hence,

$$
\begin{gathered}
\|x(t)\| \leq u(t) \leq\left(c_{1}+\frac{\eta(\bar{d}+\bar{L}) M^{\underline{v}}}{\Gamma(\nu+1)}\right) \\
\cdot\left(1+\sum_{k=1}^{\infty}(\bar{a}+\bar{b}+2 \bar{L})^{k} \cdot \frac{M^{\frac{k v}{}}}{\Gamma(k \nu+1)}\right) \leq c_{2} .
\end{gathered}
$$

As for $t \in J_{2}$, obviously, system (7) has finite-time stability. According to Definition 5, we can obtain that system (7) is finite-time stable. This completes the proof of Theorem 11.

Remark 12. Different from the research in uniqueness of solutions, the disturbance vector $w(t)$ plays a key role in studying the finite-time stability. It can be easily analyzed by (33).

When $A, B, D$ are function matrices instead of constant ones, our conclusion about the finite-time stability (Theorem 11) also can be applied to system (27). In this case, we assume that $\|A(t)\|=\bar{a},\|B(t)\|=\bar{b},\|D(t)\|=\bar{d}$ in the same proof.

Corollary 13. Given positive numbers $c_{1}, c_{2}, M$, such that $c_{1}<$ $c_{2}$, then system (27) is finite-time stable on $J_{1} \cup J_{2}$ if all the conditions in Theorem 11 hold.

\section{Example}

In this section, we will present the following two examples to illustrate our main results.

Example 14. Suppose that $v=1 / 20$, and $f(\cdot)=0.1 \sin x(t)+$ $0.1 \cos x(t-h(t))$ with $x=\left(x_{1}(t), x_{2}(t)\right)^{T}$. Consider system (7), where

$$
\begin{aligned}
& A=\left(\begin{array}{ll}
1 & 0 \\
0 & 2
\end{array}\right), \\
& B=\left(\begin{array}{ll}
2 & 3 \\
0 & 0
\end{array}\right) .
\end{aligned}
$$

We have $\|A\|=\bar{a}=4,\|B\|=\bar{b}=13$, and $\bar{L}=0.1, \Gamma(\nu) \approx$ 19.47 , which imply that the condition $0<(\bar{a}+\bar{b}+2 \bar{L}) / \Gamma(\nu)<1$ referred to in Theorem 8 holds. Therefore, the specific system (7) has a unique solution.

Remark 15. Since there are few papers researching the uniqueness of solutions for the nonlinear fractional-order difference equation with time-varying delay, one can see that all the results in $[23,25-28]$ can not directly be applicable to Example 14 to obtain the uniqueness of the solution. This implies that the results in this paper are essentially new.

Example 16. Suppose that

$$
f(\cdot)=0.1\left(\begin{array}{c}
\sqrt{x_{1}^{2}(t)+x_{1}^{2}(t-h)}+\sin x_{1} \\
\sqrt{x_{2}^{2}(t)+x_{2}^{2}(t-h)}+\sin x_{2}
\end{array}\right),
$$

and let

$$
\begin{aligned}
& A=\left(\begin{array}{cc}
0.2 & 0 \\
0 & 0.3
\end{array}\right), \\
& B=\left(\begin{array}{cc}
0 & 0 \\
0.4 & 0.2
\end{array}\right), \\
& D=\left(\begin{array}{l}
1 \\
0
\end{array}\right) .
\end{aligned}
$$

Let $M=10, w(t)=\sqrt{2}, h=1, v=0.03, \Gamma(\nu) \approx 32.79$, $\Gamma(\nu+1) \approx 0.98, a=1, \eta=1.42, \phi(t)=(0.8,0.8)^{T}$. We have $\bar{a}=0.3, \bar{b}=0.45, \bar{d}=1, \bar{L}=0.1$. One can assume that $c_{1}=1.14, c_{2} \geq 463.26,0<(\bar{a}+\bar{b}+2 \bar{L}) / \Gamma(\nu) \approx 0.97<1$. By Mathematica software, we can get

$$
\begin{aligned}
& \left(c_{1}+\frac{\eta(\bar{d}+\bar{L}) M^{\underline{v}}}{\Gamma(\nu+1)}\right) \\
& \quad\left(1+\sum_{k=1}^{\infty}(\bar{a}+\bar{b}+2 \bar{L})^{k} \cdot \frac{M^{\underline{k v}}}{\Gamma(k v+1)}\right) \approx 463.26 \\
& \leq c_{2},
\end{aligned}
$$

which implies that inequality (28) holds, and we conclude that all conditions in Theorem 11 are satisfied. Therefore, the specific system (7) is finite-time stable.

\section{Conclusion}

In this paper, we are concerned with a nonlinear fractionalorder difference equation. The addressed equation has time delay terms, which are quite different from the related references discussed in the literature [18, 23, 24, 28, 29]. The nonlinear fractional-order difference system studied in the present paper is more generalized and more practical. By applying the generalized Gronwall inequality and the definition of the finite-time stability, we employ a novel argument and the easily verifiable sufficient conditions have 
been provided to determine the uniqueness and finitetime stability of the solutions for the considered equation. Finally, two typical examples have been presented at the end of this paper to illustrate the effectiveness and feasibility of the proposed criterion. Consequently, this paper shows theoretically and numerically that some related references known in the literature can be enriched and complemented.

\section{Data Availability}

The data in this study were mainly collected via discussion during our class. Readers wishing to access these data can do so by contacting the corresponding author.

\section{Conflicts of Interest}

The authors declare that they have no conflicts of interest.

\section{Acknowledgments}

This work was supported by the National Natural Science Foundation of China (Grant No. 11471109).

\section{References}

[1] B. Kuttner, "On differences of fractional order," Proceedings of the London Mathematical Society. Third Series, vol. 7, pp. 453466, 1957.

[2] C. Goodrich and A. C. Peterson, Discrete Fractional Calculus, Springer International, New York, NY, USA, 2015.

[3] T. Abdeljawad, Q. M. Al-Mdallal, and M. . Hajji, "Arbitrary order fractional difference operators with discrete exponential kernels and applications," Discrete Dynamics in Nature and Society, Art. ID 4149320, 8 pages, 2017.

[4] T. Abdeljawad and Q. M. Al-Mdallal, "Discrete Mittag-Leffler kernel type fractional difference initial value problems and Gronwall's inequality," Journal of Computational and Applied Mathematics, vol. 339, pp. 218-230, 2018.

[5] Y. Sun and G. Li, "Finite-time stability and stabilization of networked control systems with bounded Markovian packet dropout," Discrete Dynamics in Nature and Society, Art. ID 176919, 6 pages, 2014.

[6] X. Mo, H. Niu, and Q. Lan, "Finite-time stabilization for a class of nonlinear differential-algebraic systems subject to disturbance," Discrete Dynamics in Nature and Society, Art. ID 9730312, 14 pages, 2017.

[7] Y. Xu, J. Zhang, W. Zhou, and D. Tong, "Finite-time bounded synchronization of the growing complex network with nondelayed and delayed coupling," Discrete Dynamics in Nature and Society, Art. ID 6501583, 7 pages, 2017.

[8] C. Lizama and M. Murillo-Arcila, "Maximal regularity in $l^{p}$ spaces for discrete time fractional shifted equations," Journal of Differential Equations, vol. 263, no. 6, pp. 3175-3196, 2017.

[9] T. Yu, Y. Zhong, T. Chen, and C. Chen, "Finite-time stabilization of uncertain switched positive linear systems with time-varying delays," Discrete Dynamics in Nature and Society, vol. 2015, Article ID 954782, 9 pages, 2015.

[10] T. Abdeljawad and B. Abdalla, "Monotonicity results for delta and nabla caputo and riemann fractional differences via dual identities," Filomat, vol. 31, no. 12, pp. 3671-3683, 2017.
[11] T. Abdeljawad and D. F. M. Torres, "Symmetric duality for left and right Riemann-Liouville and Caputo fractional differences," Arab Journal of Mathematical Sciences, vol. 23, pp. 157-172, 2017.

[12] I. K. Dassios and D. I. Baleanu, "Duality of singular linear systems of fractional nabla difference equations," Applied Mathematical Modelling, vol. 39, no. 14, pp. 4180-4195, 2015.

[13] F. M. Atıc1 and P. W. Eloe, "Two-point boundary value problems for finite fractional difference equations," Journal of Difference Equations and Applications, vol. 17, no. 4, pp. 445-456, 2011.

[14] G.-C. Wu, D. Baleanu, and S.-D. Zeng, "Finite-time stability of discrete fractional delay systems: Gronwall inequality and stability criterion," Communications in Nonlinear Science and Numerical Simulation, vol. 57, pp. 299-308, 2018.

[15] T. Abdeljawad, "On Riemann and Caputo fractional differences," Computers \& Mathematics with Applications, vol. 62, no. 3, pp. 1602-1611, 2011.

[16] T. Abdeljawad, J. Alzabut, and D. Baleanu, "A generalized $q$ fractional Gronwall inequality and its applications to nonlinear delay $q$-fractional difference systems," Journal of Inequalities and Applications, Paper No. 240, 13 pages, 2016.

[17] M. Wyrwas, D. Mozyrska, and E. Girejko, "Stability of discrete fractional-order nonlinear systems with the nabla Caputo difference," IFAC Proceedings Volumes, vol. 46, no. 1, pp. 167-171, 2013.

[18] G.-C. Wu, D. Baleanu, and W.-H. Luo, "Lyapunov functions for Riemann-Liouville-like fractional difference equations," Applied Mathematics and Computation, vol. 314, pp. 228-236, 2017.

[19] G.-C. Wu, D. Baleanu, and L.-L. Huang, "Novel Mittag-Leffler stability of linear fractional delay difference equations with impulse," Applied Mathematics Letters, vol. 82, pp. 71-78, 2018.

[20] Fulai Chen and Zhigang Liu, "Asymptotic Stability Results for Nonlinear Fractional Difference Equations," Journal of Applied Mathematics, vol. 2012, Article ID 879657, 14 pages, 2012.

[21] R. Abu-Saris and Q. Al-Mdallal, "On the asymptotic stability of linear system of fractional-order difference equations," Fractional Calculus and Applied Analysis, vol. 16, no. 3, pp. 613-629, 2013.

[22] D. Baleanu, G.-C. Wu, Y.-R. Bai, and F.-L. Chen, "Stability analysis of Caputo-like discrete fractional systems," Communications in Nonlinear Science and Numerical Simulation, vol. 48, pp. 520530, 2017.

[23] C. S. Goodrich, "Existence and uniqueness of solutions to a fractional difference equation with nonlocal conditions," Computers \& Mathematics with Applications, vol. 61, no. 2, pp. 191-202, 2011.

[24] C. S. Goodrich, "Continuity of solutions to discrete fractional initial value problems," Computers \& Mathematics with Applications, vol. 59, no. 11, pp. 3489-3499, 2010.

[25] L. Plociniczak and M. Switala, "Existence and uniqueness results for a time-fractional nonlinear diffusion equation," Journal of Mathematical Analysis and Applications, vol. 462, no. 2, pp. 1425-1434, 2018.

[26] Y. Zou and G. He, "On the uniqueness of solutions for a class of fractional differential equations," Applied Mathematics Letters, vol. 74, pp. 68-73, 2017.

[27] C. S. Goodrich, "On positive solutions to nonlocal fractional and integer-order difference equations," Applicable Analysis and Discrete Mathematics, vol. 5, no. 1, pp. 122-132, 2011. 
[28] F. L. Chen and Y. Zhou, "Existence and Ulam stability of solutions for discrete fractional boundary value problem," Discrete Dynamics in Nature and Society, vol. 2013, Article ID 459161, 7 pages, 2013.

[29] C. S. Goodrich, "A convexity result for fractional differences," Applied Mathematics Letters, vol. 35, no. 1, pp. 58-62, 2014. 


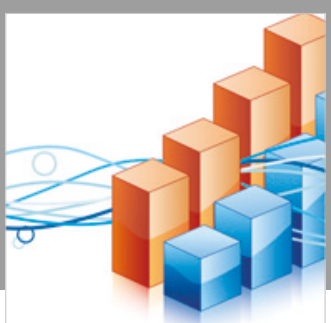

Advances in

Operations Research

\section{-n-m}
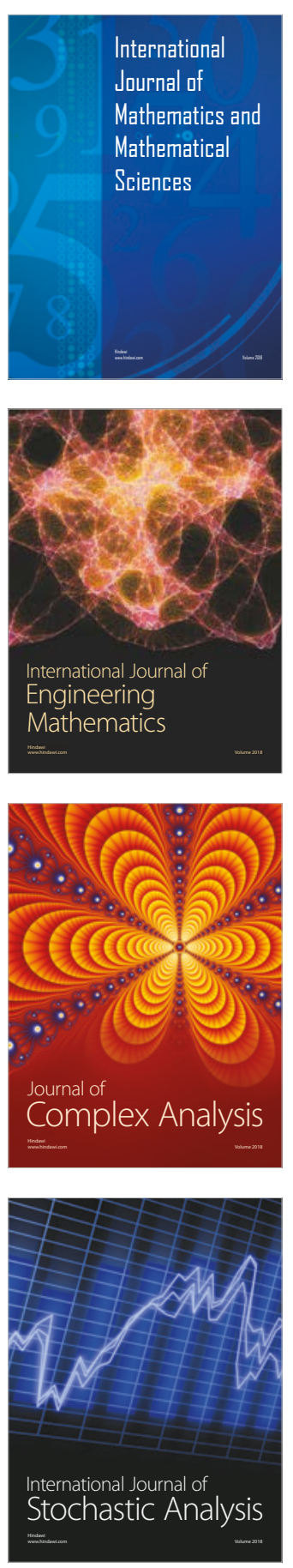
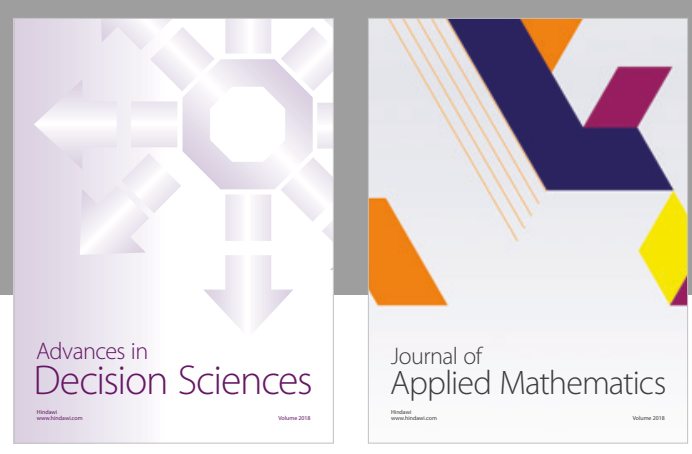

Journal of

Applied Mathematics
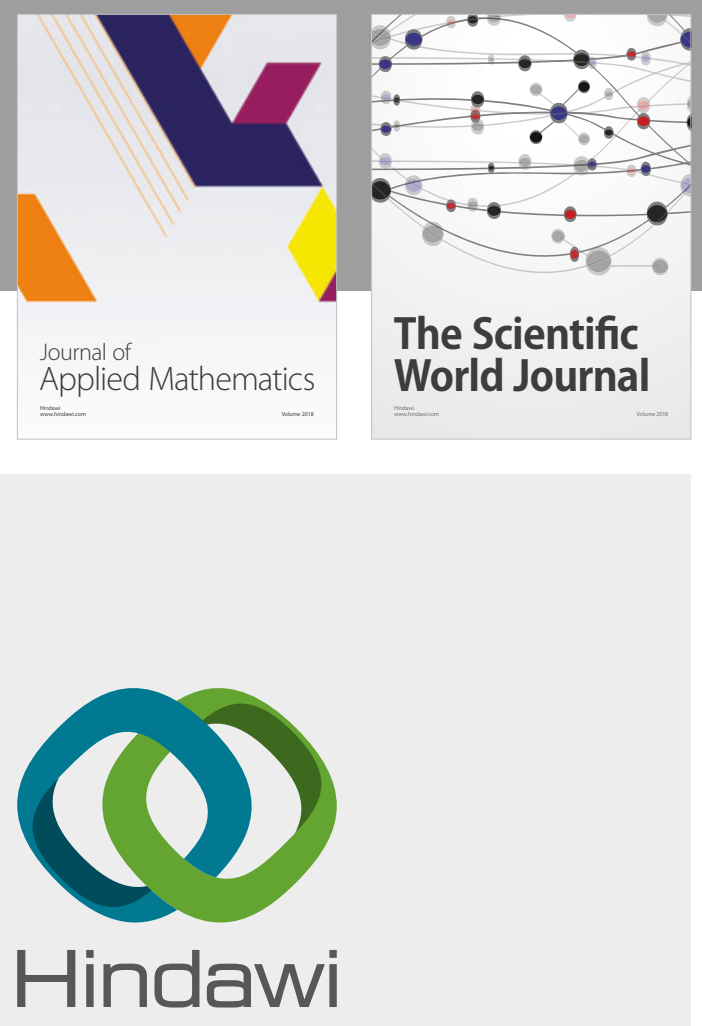

Submit your manuscripts at

www.hindawi.com

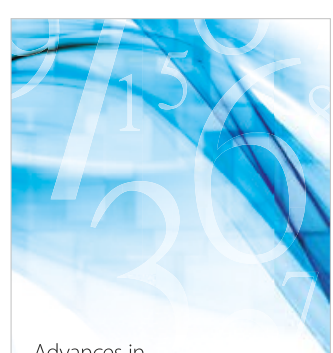

Advances in
Numerical Analysis
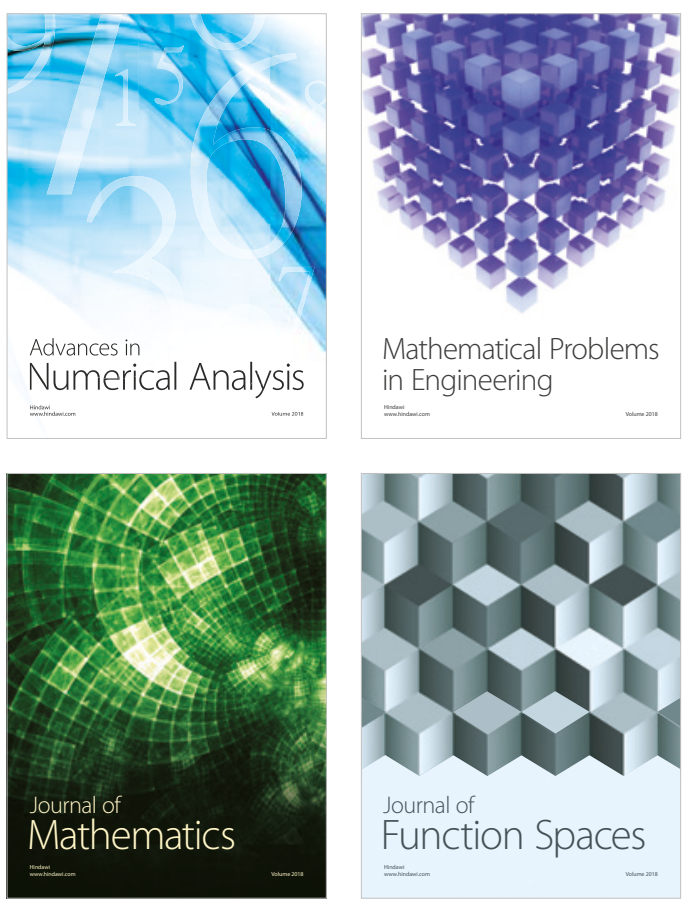

Mathematical Problems in Engineering

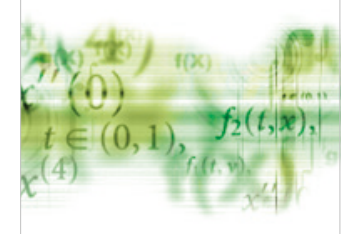

International Journal of

Differential Equations

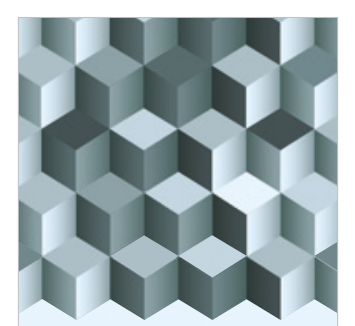

Journal of

Function Spaces

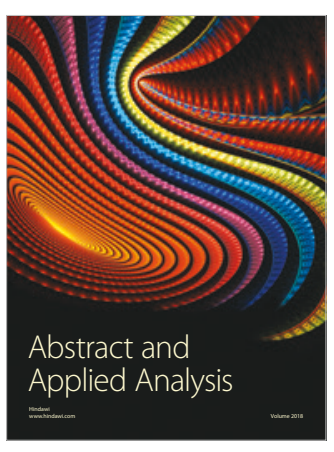

The Scientific

World Journal

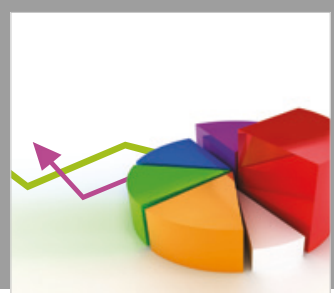

Journal of

Probability and Statistics
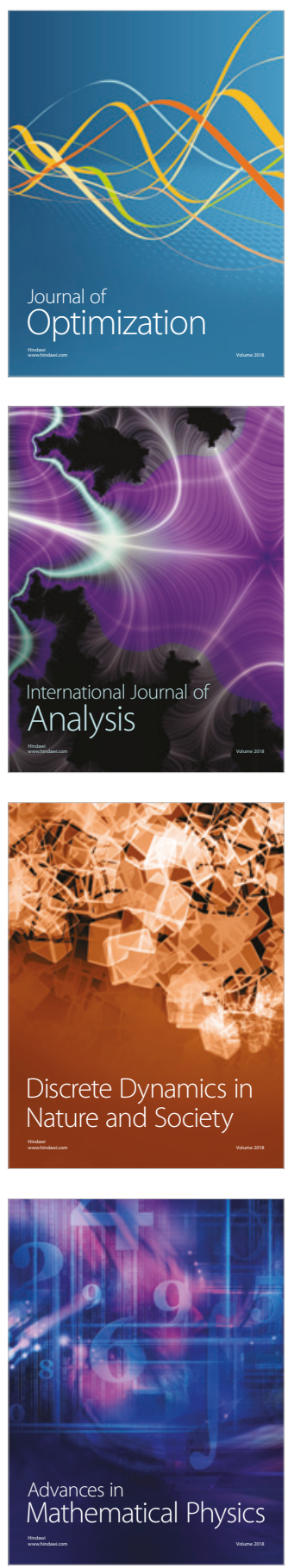